\title{
Effect of Objects Surrounding a Referent on Young Children's Interpretation of Word Meanings
}

\author{
Takahiro Tamura $^{1}$ \\ ${ }^{1}$ College of Education, Naruto University of Education, Naruto City, Tokushima, Japan \\ Correspondence: Takahiro Tamura, College of Education, Naruto University of Education, Takashima, Naruto-Cho, \\ Naruto City, 775-8502, Japan.
}

Received: November 28, 2016

Accepted: December 14, $2016 \quad$ Available online: December 23, 2016

doi:10.11114/ijsss.v5i1.1994

URL: http://dx.doi.org/10.11114/ijsss.v5i1.1994

\begin{abstract}
This study examined the effect of objects surrounding a referent on young children's interpretation of word meanings. In Study 1, 50 children aged 5 years (28 females, 22 males; mean age: 5 years 8 months, SD: 2.37) took part in a novel label task wherein they were given a novel word for a target object with its surrounding object in two conditions, namely, high ordinariness (e.g., an elephant in a cage) or low ordinariness (e.g., an elephant riding in a car). The participants in both conditions tended to interpret the novel word as a name denoting the target object and its surrounding object. In Study 2, which used the same task, 50 children aged 5 years (26 females, 24 males; mean age: 5 years 8 months, SD: 2.47) were given a novel word for a target object with its surrounding object in two conditions, namely high proximity (e.g., an elephant in a cage) or low proximity (e.g., an elephant near a tree). Participants in the high proximity condition tended to interpret the novel word as referring to a name that incorporated the target item and its surrounding object. In contrast, the participants in low proximity condition tended to interpret the novel word as referring to the name of the target object only (e.g., "elephant"). These results suggest that perceptual factors, such as the proximity of the referent and its surrounding objects, influence word learning in young children. The findings are discussed with respect to young children's word learning in everyday life.
\end{abstract}

Keywords: cognitive development, word learning, language development, context effects, objects surrounding a referent

\section{Introduction}

When young children are taught an object's name directly by adults they primarily learn the meanings of the name through ostensive definition, that is, through hearing the object's name used when the object is indicated (e.g., "This is an X."). However, learning through ostensive definition is difficult because the child must select a single correct meaning from a vast number of possibilities. In spite of this difficulty, young children learn word meanings very quickly. Many researchers have postulated cognitive constraints or biases that guide young children's initial hypotheses about the meanings of ostensively defined words to explain this learning facility (Markman and Hutchinson, 1984; Markman and Wachtel, 1988). For example, taxonomic constraints (Markman and Hutchinson, 1984) propose that young children extend noun meanings to referents that have the same taxonomic relationship. For example, when a target object was labeled novel name (e.g., dog), 4- and 5-year-olds recognized that the label applied to a taxonomic associate (e.g., cat), not a thematic associate (e.g., bone). In addition, the mutual exclusivity assumption and the whole object assumption (Markman and Wachtel, 1988) suggest that young children suppose that only one name exists for a given objects and the name given to an object refers to the whole object. After the existence of these cognitive constraints and biases were proposed, various factors that affect word learning were revealed in subsequent studies. Bloom (2000) suggested that children learn words through sophisticated cognitive abilities that exist for other purposes, that is, the ability to infer others' intentions, the ability to acquire concepts, an appreciation of syntactic structure, and certain general learning and memory abilities. In particular, utilization of the ability to infer others' intentions suggests that young children use a variety of information provided by the environment in their word learning process. Indeed, recent research demonstrates that such information plays an important role in the word learning of young children, i.e., contextually cued attention (Smith, Colunga, and Yoshida, 2010; Samuelson, Smith, Perry, and Spencer, 2011; Benitez and Smith, 2012) and pragmatic cues (Tomasello and Akhtar, 1995; Grassmann, Stracke, and Tomasello, 2009; Grassmann and Tomasello, 2010). 
In previous studies on cognitive constraint and bias, children were presented with a single target object (e.g., a dog), and told "This is an X (novel word)". Thereafter, they easily recognize that "X" referred to the target object. It has been suggested that the existence of cognitive constraints or biases are of relevance to young children's learning of word meanings in experimental settings. However, in daily life, when young children hear an object name there may be a variety of objects around the referent. If young children's word learning incorporates the variety of information provided by the environment, the process might be affected by the variety of objects around the referent. In recent studies, the process of object-name learning has been examined when various objects surround the referent (i.e., Horst, Scott, and Pollard, 2010; Perry, Samuelson, Malloy, and Schiffer, 2010; Axelsson, Perry, Scott, and Horst, 2016); however, these studies focused on the learning and retention of one name for a target object. A few studies have addressed whether young children interpret a name applied to a referent as including its surrounding objects, if they learn an object name when other objects are present around the referent. That young children may interpret a referent name as including its surrounding objects suggests that the existence of objects surrounding a referent per se influences the word-meaning acquisition of young children. Studies that have addressed this issue are described next.

Hall and Waxman (1993) found that young children interpreted a novel name applied to a stuffed bear riding in a car as referring to an object name (i.e., bear) when they were told "This is a murvil." The name was restricted to a target object riding in a car (i.e., passenger) if they heard the instruction "This is a murvil because it is riding in a car." The latter result suggests that young children may interpret a name applied to a referent ("a bear") as including objects surrounding the referent ("a car") given specific information provided by others. Similarly, Tamura (1997) examined young children's interpretation of novel words. In the experiment, when the experimenter provided a novel word for targets that were in a cage (e.g., "This is a keku."), the participants tended to qualify the word as referring only to similarly restricted situations (i.e., when the referent was in a cage). This result of Tamura (1997) conflicts with the former result of Hall and Waxman (1993). Although a novel word was used to label a target object with surrounding objects in both Hall and Waxman (1993) and Tamura (1997) in the same way, their participants interpreted the meaning of a novel word differently. What caused the difference between the results of these two studies? In response, Tamura (2005) noted procedural differences between Hall and Waxman (1993) and Tamura (1997), and examined the effects of such differences on young children's interpretation of word meanings. Tamura (2005) highlighted the diversity of the surrounding objects in the aforementioned studied. That is, there was a variety of objects around the target object in Hall and Waxman (1993), whereas there was single object near the target object in Tamura (1997). Further, the target objects in Hall and Waxman (1993) were relatively large as compared with their surroundings, whereas the target objects in Tamura (1997) were relatively small; of the surrounding objects, only a cage approximated the size of the target object. In Experiment 1 of Tamura (2005), in the high diversity condition, the experimenter labeled with a novel word a target object (an elephant) that was in a cage with many objects (other animals and tools) around it. In contrast, in the low diversity condition, the experimenter labeled with a novel word a target object that was alone in a cage. As a result, the participants in the high-diversity condition tended to qualify the word meaning with an object's name (an elephant), whereas the participants in the low-diversity condition tended to qualify the word meaning as applying only when the target was similarly restricted by its surrounding objects (i.e., in a cage). In Experiment 2 of Tamura (2005), in the large-dimension condition, the experimenter labeled with a novel word a target object that was in a large cage. Conversely, in the small-dimension condition, the experimenter labeled with a novel word a target object that was in a small cage. The height and width of the large cage were three times those of the small cage. As a result, the participants in the large-dimension condition tended to qualify the word as denoting the object's name, whereas the participants in the small-dimension condition tended to qualify the word as applying only to objects similarly restricted by surrounding objects. These results clearly show that the diversity and dimensions of surrounding objects affect the qualification of word meanings that include an object's name.

Tamura (2005) considered external aspects of the relationship between the referent and its surrounding objects. However, could factors other than that examined in Tamura (2005) effect the interpretation of word meanings by young children? Recall the experimental stimuli in Hall and Waxman (1993) and Tamura (1997). The target item was a bear riding in a car in Hall and Waxman (1993), whereas it was an elephant in a cage in Tamura (2005). A bear riding in a car is unusual, i.e. it has a low ordinariness; in contrast, an elephant in a cage has a high ordinariness. This ordinariness defines difference in the quality of the relationship between the target item and the surrounding objects in Hall and Waxman (1993) versus Tamura (1997), and this difference may have affected the children's interpretation of word meanings.

The current study examined the effects of the quality of the relationship between the target item and its surrounding object on young children's interpretation of word meanings. Few previous studies have focused on the relationship between a referent and objects surrounding the referent. Meints, Plunkett, Harris, and Dimmock (2004) investigated how different background contexts influence infants' recognition of an object's name, by focusing on the typicality of 
the relationship between the referent and background. However, they did not address the relationship between the referent and specifics of the surrounding objects. To better understand how learning might be optimized, it is necessary to clarify the effects of such relationships.

We first focus on ordinariness of the relationship between the target item and its surrounding objects. The young children in Tamura's study (1997) tended to have qualified the word meanings as applying to situations similarly restricted by the surroundings due to the high ordinariness in the relationship between the target item and its surrounding objects. In contrast, in Hall and Waxman (1993), young children did not tend to restrict the word meaning according to the surroundings due to the low ordinariness in the relationship between the target item and its surrounding objects. In Study 1, we examined the pure effects of ordinariness in the relationship between the target item and its surrounding objects on young children's interpretation of word meanings, while controlling for the effects of the diversity and relative dimensions of the surrounding objects.

Here, we targeted 5 year-old children as participants because children of this age have more life experience than younger children, and thus clearer knowledge of what constitutes an "ordinary" situation. Therefore, it is possible to examine the effects of ordinariness efficiently via 5 year-old children.

If young children's interpretation of word meanings is affected by the ordinariness of the relationship between the target item and its surrounding objects, the number of participants who interpret the novel word as referring to the name of only the target object should be greater in low-ordinariness conditions than in high-ordinariness conditions.

\section{Study 1}

\section{Method}

\subsection{Participants}

Fifty public kindergarten children ( 22 boys, 28 girls, mean age: 5 years 8 months, age range: 5 years 3 months to 5 years 10 months) in Japan participated in this study. All belonged to middle-class families and were monolingual Japanese speakers, with no developmental delay in intellectual or language abilities.Children were randomly assigned to one of two conditions such that each condition was equivalent in terms of the ages and sex ratios of its participants. Permission to participate freely in this study was obtained from all children's parents. All children participated in this study without being forced. In addition, we obeyed the ethical principles of the Japanese Association of Educational Psychology in our experimental implementation of the following two studies. Furthermore, the experimental procedure did not present ethical issues to the parents of participants or the kindergarten teachers.

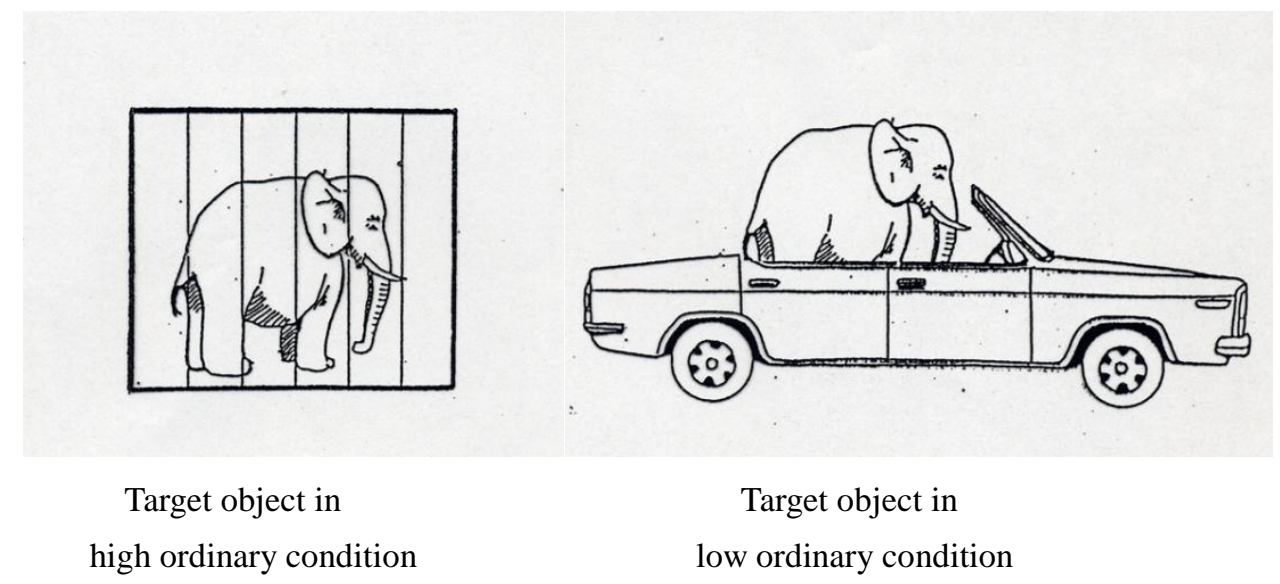

Figure 1. A sample of target objects in Study 1

\subsection{Materials}

There were two conditions in which a target object was labeled with a novel word. In the high ordinariness condition, participants were presented with an ordinary situation involving the target object (i.e., they were shown an elephant in a cage as the target object). In the low ordinariness condition, participants were presented with an extraordinary situation involving the target object (i.e., they were shown an elephant riding in a car as the target object). The degree of ordinariness of the situation in these conditions was examined by asking 20 university students to rate the ordinariness (the highest possible score was 5, and the lowest one was 1). The mean rating for an elephant in a cage was 3.8(SD: 0.52 ), and 1.5(SD: 0.51) for an elephant in a car. University student were chosen as participants for this study component because it is difficult for 5-year-old children to judge and verbally report the degree of ordinariness of a specific situation. For example, young children may consider an animal riding in a car to be an ordinary situation, given 
that this is often seen in children's picture books. However, 5-year-old children generalize differently the internal attributes of bears as personified in a picture book and real bears in a photograph (Waxman, Herrmann, Woodring, and Medin, 2014). This illustrates that 5-year-old children can distinguish the ordinariness of the real world and the world of a picture book, and the control for ordinariness in this study is therefore appropriate. Figure1 shows a sample of target objects in Study 1.

Participants were presented with 13 object choices to assess their interpretation of the novel word applied to the target object. The choices in the high ordinariness condition were two elephants, two tigers, a pigeon, two elephants in a cage, two tigers in a cage, a pigeon in a cage, a pencil, a pair of scissors, and an empty cage. The choices in low ordinariness condition were two elephants, two tigers, a pigeon, two elephants riding a car, two tigers riding a car, a pigeon riding a car, a pencil and a pair of scissors, and a car without an animal inside. The elephant in the choice objects was equivalent to the target object in terms of its basic category (i.e. elephant). The tiger items were equivalent to the target object in terms of their superordinate category (i.e., mammal). The pigeon items were equivalent to the target object in terms of a superordinate category (i.e., animal). The pencil and scissors items were unrelated to the animal category. The cage or empty car was the object that surrounded the target objects. All items were represented as a black line drawing on a $7 \mathrm{~cm}$ $\times 7 \mathrm{~cm}$ white sheet of paper. We used two-dimensional (picture) probes so that participants paid attention to only the ordinariness in relationship between the target object and the objects surrounding the target object, avoiding the influence of additional factors that can be present when using three-dimensional probes.

\subsection{Procedure}

Children were randomly assigned to the high ordinariness or low ordinariness condition and tested individually in a kindergarten room.

High ordinariness condition. The participants in this condition were shown the picture of an elephant in a cage as the target item and instructed, "Let's play a game with a foreign language." by the experimenter. Then, the experimenter pointed at the elephant in the picture and said, "Look at this. This is called a keku (novel word) in a foreign language." Subsequently, the experimenter presented 13 pictures of the choice items for the high ordinariness condition, one by one in random order and asked for each "Do you think that this is called a keku in the foreign language?" Successive questions addressed different basic-level categories (Rosch, 1978) to avoid focusing the participants' attention on particular category. That is, if the subject of one question was "elephant," then the subject of the next question would not be "elephant," and would be "tiger," "pigeon," or "pencil". The order in which questions were presented was different for each child. The participants answered "Yes" or "No" for each choice item. We used a binary choice ("yes" or "no") to determine whether participants judged that the novel label certainly did or did not apply to each item.

Low ordinariness condition. The participants in this condition were shown the picture of an elephant riding in a car as the target item and the 13 choice items for the low ordinariness condition. The procedure was the same as the high ordinariness condition, except for the target item and choice items.

The assessment of the subject's interpretation of novel word meaning. If participants interpreted the novel word to refer to "elephant", they selected only the elephant items, irrespective of whether or not a cage or a car was present. In contrast, if participants interpreted the novel word to refer to "mammal", they selected only the elephant and tiger, irrespective of whether or not a cage or a car was present. Further, the novel word was interpreted as referring to "animal", participants selected only elephant, tiger, and pigeon items, regardless of whether a cage or a car was present. The cage and car items were contained in the choice objects so that we could assess whether the novel word was interpreted as referring to a name restricted by the surrounding situation. If participants selected the object in a cage or a car as the referent of novel word applied to the target object, they interpreted the novel word as referring to a name restricted by the situation.

\subsection{Analysis}

Children's choices of pictures were classified into the following three patterns: object name patterns, in which regardless of the presence or absence of a cage, all of the elephant items were chosen but no other items; name restricted surrounding situation patterns, in which all elephant in a cage items were chosen, but no other item; and other patterns, in which included patterns that were not categorized as object name patterns or name restricted surrounding situation patterns. We defined three classification patterns based on the choice of the elephant because it was easy for the young children to recognize a basic level name ("an elephant") as the name that labeled an object. We assume that the participants who used the object name patterns interpreted the novel word as "elephant". To examine the effects of the existence of surrounding objects on the interpretation of novel word, Fisher's exact test was performed to test for differences in the number of children who used each choice pattern. When examining about a choice pattern, the number of people of other patterns was made included. 


\section{Results and Discussion}

Table 1 shows the numbers and percentages of children within each condition whose choices were characterized by each pattern. The test was non- significant for all three patterns. This result indicates that the young children tended to interpret the novel word as referring to a name restricted by the situation, regardless of ordinariness of the relationship between the referent and its surrounding object. This suggests that the interpretation of a novel word was influenced little by the relationship between the referent and its surrounding object, such as the ordinariness of the relationship. That is, the interpretation of word meanings was not markedly influenced by cognitive factors that involved knowledge of the situation encompassing the referent. The hypothesis of Study 1 was therefore rejected. Vlach and Sandhofer (2011) reported that when the background context (the color of a piece of cloth) of an object changed, children under the age of 4 years did not recognize it as an object belonging to the same category and did not apply the same name to such objects, even if they were actually the same object. Thus, the interpretation of word meanings by young children is affected by subtle aspects of the context surrounding the reference. The results of Study 1 may reflect that objects surrounding a referent influenced the interpretation of word meanings by young children, as examples of such context.

Therefore, what factors influence young children's interpretation of word meanings? In this study, young children tended to interpret the word meanings as referring to a name restricted by the situation in both conditions. However, young children do not interpret word meanings in such a manner during the process of practical language acquisition. Specifically, the interpretation of word meanings as referring to a name restricted by the attendant situation is uncommon in actual language acquisition. In reality, to learn the correct meaning of a word, young children must interpret word meanings without restricting the meanings to a specific situation. That is, there is likely a factor that encourages young children to interpret word meanings without situation-dependent restrictions. Reconsidering the results of Tamura (2005), it was demonstrated that the diversity and dimensions of the objects surrounding the referent engendered young children interpreting word meanings without situational restrictions. These factors concern perception rather than cognitions based on prior knowledge. In young children's actual language acquisition, such perceptual factors might influence learning the correct word meanings. As such, it is necessary to consider the experimental design of Study 1 with actual word learning, while considering perceptual factors. In Study 1, the referent lay in close proximity to its surrounding objects. However, during word learning in real life, the referent does not always lie in close proximity to its surrounding specific objects. Young children may be sensitive to the proximity of the referent and its surrounding objects, and this proximity may influence the interpretation of word meanings pertaining to the referent during actual word learning. Indeed, it has been clarified that the spatial distance between a referent and objects surrounding a referent influence the word learning of two- year-old children (Axelsson, Perry, Scott, and Horst, 2016). This suggests that perceptual factors influence the word learning of young children.

Therefore, in Study 2, we examined the pure effect of proximity between the target item and the surrounding objects on young children's interpretation of word meanings, while controlling for the effects of the diversity and relative dimensions of the surrounding objects.

In Study 2, we targeted 5-year-old children as participants, as in Study 1. It appears that 5-year-old children are more sensitive to the proximity of a referent and its surrounding objects than younger children, because of development of perceptual processes (Zaporozhets, 1965).

If the interpretation of word meanings of young children is affected by proximity in the relationship between the target item and its surrounding objects, participants would tend to interpret a novel word as referring to the name of the target object only in low proximity rather than high proximity situations.

Table1. Number and Percentage of Children within Each Condition (Whose Choices were Characterized by Each Pattern in Study 1.)

\begin{tabular}{lrrrr}
\hline Pattern & \multicolumn{2}{c}{ High Ordinariness } & \multicolumn{2}{c}{ Low Ordinariness } \\
& $\mathrm{n}$ & $\%$ & $\mathrm{n}$ & $\%$ \\
\hline Object Name & 16 & 64 & 14 & 56 \\
Name Restricted Surrounding Situation & 7 & 28 & 10 & 40 \\
Other & 2 & 8 & 1 & 4 \\
\hline
\end{tabular}

\section{Study 2}

Study 1 suggested that knowledge-based cognitive factors influenced minimally children's interpretation of word meanings. Furthermore, it was suggested that perceptual factors might also have an influence, consistent with previous research (i.e., Tamura, 2005). In this second study, we examined the influence of perceptual factors on young children's interpretation of word meanings, again for referents with surrounding objects. We focused on the proximity between the referent and its surrounding objects. We expected that young children would interpret word meanings without 
situational restrictions if the proximity between the referent and its surrounding objects was low. This is because the proximity between the referent and its surrounding objects is typically low during real-life word learning, and young children usually interpret word meanings without restrictions in such a real-world. That is, referents exist in various contexts in real world, so they are not always in close proximity with other specific objects. In contrast, in the light of the discoveries of Study 1 and Tamura (1997), we expected that young children would likely interpret word meanings as restricted by the situation if the proximity between the referent and its surrounding objects was high.

\section{Method}

\subsection{Participants}

Fifty public kindergarten children (24 boys, 26 girls; mean age: 5 years 8 months, age range: 5 years 4 months to 5 years 11 months) in Japan participated in this study. They all belonged to middle-class families were monolingual Japanese speakers, and none had delayed intellectual or language development. Children were randomly assigned to one of two conditions such that each condition was equivalent in terms of ages and sex ratios. Permission to participate freely in this study was obtained from all children's parents. All children participated in this study without being forced.

\subsection{Materials}

There were two conditions in which a target object was labeled with a novel word. In the high proximity condition, participants were presented with a target object that was closed to the surrounding objects (i.e., they were shown an elephant in a cage as the target object). In the low proximity condition, participants were presented with a target object that was not close to the surrounding objects (i.e., they were shown an elephant near a tree as the target object). The proximity between the target object and its surrounding objects was quantified by 20 university students who rated proximity of the objects (The most proximal rating was 5, and the least proximal rating was 1 ). The mean of rating for an elephant in a cage was 3.9(SD: 0.55), whereas as an elephant near a tree was given a mean rating of 1.4(SD: 0.50). Figure1 shows a sample of target objects in Study 2.

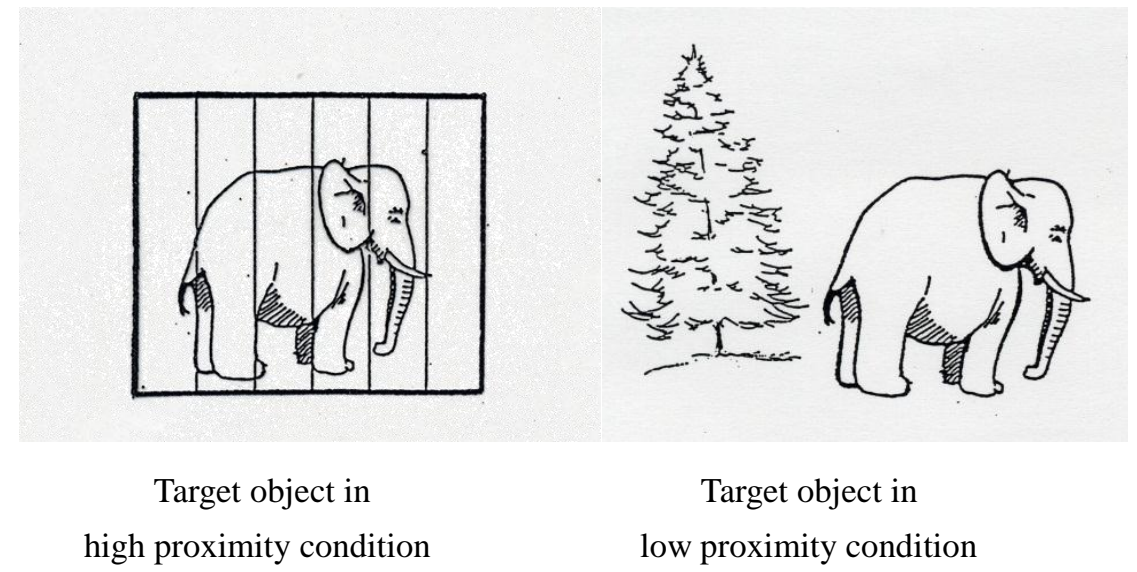

Figure 2. A sample of target objects in Study 2

Participants were presented with a choice of 13 objects to assess their interpretation of the novel name word applied to the target object. The objects in the high proximity condition were the same as those used in the high ordinariness condition of Study 1. The objects in the low proximity condition were two elephants, two tigers, and a pigeon; and two elephants near a tree, two tigers near a tree, and a pigeon near a tree, a pencil and a pair of scissors, and a tree. Each choice corresponded to a conceptual level of interpretation of the word meanings as described for Study 1 . All items were represented as a black line drawing on a $7 \mathrm{~cm} \times 7 \mathrm{~cm}$ white sheet of paper, as in Study 1. We used two-dimensional (picture) probes to ensure participants paid attention to only the proximity in the relationship between the target object and the surrounding objects, and to avoid the influence of various factors that can be present in three-dimensional probes (e.g., the difference in proximity between targets and other objects is contingent on the viewing angle).

\subsection{Procedure}

High Proximity Condition The participants in this condition were shown the picture of an elephant in a cage as the target item and instructed, "Let's play a game with a foreign language." by the experimenter. Then, the experimenter pointed at the elephant in the picture and said "Look at this. It is called a wamo (novel word) in a foreign language." After giving these instructions, the Experimenter presented 13 pictures of the choice items one-by-one in random order and asked for each "Do you think that this is called a wamo in the foreign language?" The participants answered "yes" or "no" for each choice item. The order of presentation of questions was same as in Study 1. 
Low Proximity Condition The participants were shown the picture of an elephant near a tree as the target item. Otherwise, the procedure was identical to the high proximity condition.

\subsection{Analysis}

Children's choices of pictures were classified into the following three patterns: object name patterns, in which, regardless of the presence or absence of a cage or a tree, all of the elephant items were chosen, but no other items; name restricted to the situation patterns, in which all items showing an elephant in a cage or near a tree were chosen, but no other item; and other patterns, in which consisted of all patterns that were not object name patterns or name restricted to situation patterns. We assume that the participants who responded with the object name patterns interpreted the novel word as "elephant". To examine the effects of proximity between the referent and the surrounding objects on interpretation of the novel word, Fisher's exact test was performed to assess the number of children who used each choice pattern. When examining about a choice pattern, the number of people of other patterns was made included.

\section{Results and Discussion}

Table 2 shows the numbers and percentages of children within each condition whose choices were characterized by each pattern. The number of participants who chose the object name patterns was greater in low proximity condition than in the high proximity condition $(\mathrm{p}<.0041)$. In contrast, the number of participants who exhibited name restricted to the situation patterns was greater in the high proximity condition than in the low proximity condition $(\mathrm{p}<.0031)$. There was no significant difference between the two conditions with respect to the number of the other patterns. These results indicate that young children tended to interpret the novel word as referring to a name restricted by the situation if the proximity between the referent and its surrounding objects was high. Conversely, they tended to interpret the novel word as referring to a name without being restricted by the situation if the proximity between the referent and its surrounding objects was low. These results suggest that perceptual factors such as the proximity of a referent and its surrounding objects, influenced the interpretation of word meanings in the young children tested and thus the hypothesis of Study 2 was supported.

Table 2. Number and Percentage of Children within Each Condition (Whose Choices were Characterized by Each Pattern in Study 2.)

\begin{tabular}{lrrrr}
\hline Pattern & High & Proximity & Low & Proximity \\
& $\mathrm{n}$ & $\%$ & $\mathrm{n}$ & $\%$ \\
\hline Object Name & 8 & 32 & 19 & 76 \\
Name Restricted Surrounding Situation & 15 & 60 & 4 & 16 \\
Other & 2 & 8 & 2 & 8 \\
\hline
\end{tabular}

\section{General Discussion}

The two studies clearly show that perceptual factors such as the proximity of a referent and its surrounding objects, influence language learning in young children, rather than cognitive factors based on preexisting knowledge, such as the ordinariness of the relationship between the referent and its surrounding objects. That young children are affected by perceptual factors during actual language learning suggests that we must recapture the impact of linguistic cognitive constraints and bias in experimental studies. Here, we found that young children tended to interpret the meaning of a word as including the referent and its surrounding objects contingent on the perceptual factors. That young children may recognize the name of objects, including plural objects in some cases, suggests that word meanings are not guided by mutual exclusivity and the whole-object assumption (Markman and Wachtel, 1988). This is because mutual exclusivity and the whole-object assumption posit that young children assume a word meaning that corresponds to the name of one of the objects present. Furthermore, taxonomic constraints suggest that the meanings of a name given to an object corresponds to the taxonomic category to which the object belongs, which is inconsistent with observations that young children recognize that a name can apply to multiple objects that belong to different categories, such as when they observe a wearing an animal collar and are told, "This is a dog.". In the same case, in adulthood, the name of "a dog" might be interpreted as meaning only a dog with a collar. This interpretation of word meanings in young children is not derived from taxonomic constraints, mutual exclusivity, or the whole object assumption. That is, in real-world situations in which a variety of objects exist around a referent, we must consider the involvement of different factors (e.g., perceptual) that influence the word learning process.

It is necessary to recognize the limitations of these studies. First, there are issues regarding the assessment of the quality of the relationship between a reference and its surrounding objects. In this study, university students (i.e., adults) assessed ordinariness and proximity because it was difficult for young children to make these assessments in response to a verbal question. However, it is possible that adults' and young children's assessments are not completely congruent. In future research, it will be necessary to devise a method that allows young children to assess the quality of the 
relationship between a referent and objects surrounding a referent. In addition, the age of participants may be problematic. In this study, 5-year-old children were chosen as participants because they have a certain degree of knowledge regarding the ordinariness of a referent and sensitivity to proximity between the referent and other objects. However, 5-year-olds might have used meta-linguistic abilities unavailable to younger children when we asked them to imagine a foreign-language related situation in the procedure of the two studies. In future studies, it would be advisable to remove the influence of meta-linguistic abilities to the extent possible, such as by using a procedure wherein a novel name is used to label a novel object.

Further research must examine systematically the effects of the factors that occur in real-world word learning, in addition to factors involved in the linguistic cognitive constraints and biases. This study made clear that perceptual factors, such as the proximity between the referent and its surrounding objects, influence real-world word learning in young children. Factors other than perceptual proximity may be involved in word learning in young children and future studies must consider on perceptual factors other than proximity.

\section{References}

Axelsson, E. L., Perry, L. K., Scott, E. J., \& Horst, J. S. (2016). Near or far: The effect of spatial distance and vocabulary knowledge on word learning. Acta Psychologica, 163, 81-87. https://doi.org/10.1016/j.actpsy.2015.11.006

Benitez, V, L., \& Smith, L, B. (2012). Predictable locations aid early object name learning. Cognition, 125, 339-352. https://doi.org/10.1016/j.cognition.2012.08.006

Bloom, P. (2000). How children learn the meaning of words? Cambridge, MA: MIT Press.

Grassmann, S., \& Tomasello, M. (2010). Young children follow pointing over words in interpreting acts of reference. Developmental Science, 13(1), 252-263. https://doi.org/10.1111/j.1467-7687.2009.00871.x

Grassmann, S., Stracke, M., \& Tomasello, M. (2009). Two-year-olds exclude novel objects as potential referents of novel words based on pragmatics. Cognition, 112(3), 488-493. https://doi.org/10.1016/j.cognition.2009.06.010

Hall, D. J., \& Waxman, S. (1993). Assumptions about word meaning: Individuation and basic-level kinds. Child Development, 64, 1550-1570. https://doi.org/10.2307/1131552

Horst, J. S., Scott, E. J., \& Pollard, J. A. (2010). The role of competition in word learning via referent selection. Developmental Science, 13(5), 706-713. https://doi.org/10.1111/j.1467-7687.2009.00926.x

Markman, E. M., \& Hutchinson, J. E. (1984). Children's sensitivity to constraints on word meaning: Taxonomic versus thematic relation. Cognitive Psychology, 16, 1-27. https://doi.org/10.1016/0010-0285(84)90002-1

Markman, E. M., \& Wachtel, G. F. (1988). Children's use of mutual exclusivity to constrain the meanings of words. Cognitive Psychology, 20, 121-157. https://doi.org/10.1016/0010-0285(88)90017-5

Meints, K., Plunkett, K., Harris, P. L., \& Dimmock, D. (2004). The cow on the high street: Effects of background context on early naming. Cognitive Development, 19(3), 275-290. https://doi.org/10.1016/j.cogdev.2004.03.004

Perry, L. K., Samuelson, L. K., Malloy, L. M., \& Schiffer, R. N. (2010). Learn locally, think globally: Exemplar variability supports higher-order generalization and word learning. Psychological Science, 21(12), 1894-1902. https://doi.org/10.1177/0956797610389189

Rosch, E. (1978). Principles of categorization. In Rosch, E. and Lloyd, B. B. (Eds.). Cognition and categorization. Hillsdale, NJ: Erlbaum, 27-48.

Samuelson, L. K., Smith, L. B., Perry, L, K., \& Spencer, J. P. (2011). Grounding word learning in space. PloS One, 6(12). http://dx.doi.org-101371/journal.pone.0028095

Smith, L. B., Colunga, E., \& Yoshida, H. (2010). Knowledge as process: Contextually cued attention and early word learning. Cognitive Science, 34, 1287-1314. https://doi.org/10.1111/j.1551-6709.2010.01130.x

Tamura, T. (1997). Effects of object's surrounding situation on qualifying word meaning in young children.] [Japanese Journal of Educational Psychology, 45, 474-481. [In Japanese with English abstract] https://doi.org/10.5926/jjep1953.45.4_474

Tamura, T. (2005). The effects of the diversity and relative dimension of the peripheral objects on the meanings of the qualifying word for young children. The Japanese Journal of Psychology, 75, 487-494. [In Japanese with English abstract] https://doi.org/10.4992/jjpsy.75.487

Tomasello, M., \& Akhtar, N. (1995). Two-year-olds use pragmatic cue to differentiate reference to objects and actions. Cognitive Development, 10, 201-224. https://doi.org/10.1016/0885-2014(95)90009-8

Vlach, H. A., \& Sandhofer, C. M. (2011). Developmental differences in children's context-dependent word learning. 
Journal of Experimental Child Psychology, 108(2), 394-401. https://doi.org/10.1016/j.jecp.2010.09.011

Waxman, S. R., Herrmann, P., Woodring, J., \& Medin, D. (2014). Humans (really) are animals: Picture-book reading influences 5-year-old urban children's construal of the relation between humans and non-human animals. Frontiers in Psychology, 5, 172. https://doi.org/10.3389/fpsyg.2014.00172

Zporozhets, A. V. (1965). The development of perception in the preschool child. In P.H. Mussen (Ed.). European research in cognitive development (Monographs of the Society for Research in Child Development, Vol. 30), 82-101. https://doi.org/10.2307/1165778

\section{Copyrights}

Copyright for this article is retained by the author(s), with first publication rights granted to the journal.

This is an open-access article distributed under the terms and conditions of the Creative Commons Attribution license which permits unrestricted use, distribution, and reproduction in any medium, provided the original work is properly cited. 